\title{
Perioperative use of statins in noncardiac surgery
}

\author{
YC Chan' \\ SW Cheng' \\ MG Irwin ${ }^{2}$ \\ 'Division of Vascular Surgery and \\ ${ }^{2}$ Anaesthesiology, University of Hong \\ Kong Medical Centre, South Wing, \\ I4th Floor K Block, Queen Mary \\ Hospital, Hong Kong, China
}

\begin{abstract}
Background: Statins (3-hydroxy-3-methylglutaryl coenzyme A reductase inhibitors) can significantly decrease cardiovascular mortality and morbidity, irrespective of the patients' cholesterol status. This paper reviews the effects of perioperative statin therapy in patients undergoing noncardiac surgery.

Method: A systematic literature review was undertaken of all published literature on this subject using Medline and cross-referenced. All published relevant papers on the perioperative use of statins were used.

Results: Perioperative statin therapy is associated with a lower perioperative morbidity and mortality in patients undergoing elective or emergency surgery. The effects are due to a combination of lipid-lowering and pleiotropic properties of statins.

Conclusion: Ideally a large scale multi-centre randomized controlled trial of perioperative statin therapy should be performed but this may be difficult to conduct since there is already overwhelming evidence in the literature to suggest perioperative cardiovascular protective properties. Statins may still be under-prescribed in surgical patients.
\end{abstract}

Keywords: perioperative, cardiac complications, morbidity, mortality, outcome, statins, vascular, noncardiac surgery

\section{Perioperative cardiac complications}

Cardiac complications are a major cause of perioperative and late morbidity and mortality, especially in patients undergoing vascular surgery (Priebe 2004). This is particularly related to underlying ischemic heart disease, which is frequently asymptomatic and the prevalence of which is increasing in many countries. Since the risk factors for coronary artery disease and peripheral vascular disease are the same, patients undergoing vascular surgical procedures are at particularly high risk in developing perioperative cardiac complications (Ashton et al 1993; Kertai et al 2006). A recent Vascular Anaesthesia Society audit identified a perioperative mortality rate of $7.3 \%$ in patients undergoing infrarenal aortic surgery with most of these deaths attributable to cardiac events (Bayly et al 2001). Unfortunately despite an improvement in the safety of modern anesthesia, better drugs and advances in perioperative patient monitoring, the mortality from myocardial infarction after noncardiac surgery is still high, and is believed to be $10 \%$ to $15 \%$ (Adesanya et al 2006). In a cohort of 323 patients with a cardiac history who underwent noncardiac surgery, Badner and colleagues (1998) showed that $18(5.6 \%)$ patients developed postoperative myocardial infarction with electrocardiogram and enzyme (creatinine kinase and troponin-T) changes, but only 3 of these were clinically apparent with central chest pain. In this cohort of patients, the great majority of myocardial infarctions occurred within the first 3 days postoperatively (Badner et al 1998). It may be difficult to assess the true incidence of postoperative myocardial infarction, as it is dependent on the specificity and sensitivity of the method of diagnosis (Gibson et al 2007), and the fact that some are silent clinically (Pasternack et al 1989). In a prospective study involving 444 patients with or at high risk for coronary artery disease who underwent elective noncardiac surgery, Mangano 
and colleagues (1992) found that postoperative myocardial ischemia increased the odds for long-term (30 days to 2 years after surgery) cardiac events (unstable angina, nonfatal myocardial infarction, cardiac death, surgical coronary revascularization) by 2.2-fold. Timing of surgery following myocardial infarction has some importance, although risk is related more to the functional status of the ventricles than time period since the myocardial infarction. At less than 6 weeks the risk is relatively high as the damaged myocardium is still healing. There appears then to be an intermediate risk period up to 3 months but, in uncomplicated cases, no benefit in delaying surgery beyond this (Eagle et al 1997).

The pathophysiology of reversible myocardial ischemia or perioperative myocardial infarction is not entirely clear, and is likely to be multifactorial. The main cause is due to rupturing of the unstable coronary atherosclerotic plaque, leading to thrombus formation, arterial stenosis and occlusion, ischemia of the cardiac muscle which may subsequently lead to myocardial infarction if this is prolonged (Priebe 2004; Cohen and Aretz 1999; Zaman et al 2000; Schouten et al 2006a). Surgical stress imposes extra myocardial workload due to local and systemic effects, such as perioperative blood loss, tachycardia, induction of catecholamines and glucocorticoids, tachycardia, blood pressure lability and pain. Patients with cardiac disease are particularly susceptible and since many of these patients are not symptomatic, it is important to try to identify those at risk and institute risk reduction strategies.

Careful preoperative evaluation can help to determine patients at particular risk, although many costly, potentially hazardous cardiac investigations are probably unnecessary and have poor predictive value. In a study of 32 patients who died within 30 days of vascular surgery, only half had evidence of ischemia on dobutamine stress echocardiography; and in $36 \%$ of the patients dobutamine stress echocardiogram did not predict the location of the infarct (Poldermans et al 2001). Many of the proposed surgeries are urgent and it may not be appropriate to undertake coronary revascularization. Furthermore there is increasing evidence that percutaneous coronary angioplasty and stenting may not be helpful in many patients (Boden et al 2007) and even place some at higher risk. This is probably because risk of myocardial infarction is related more to atheromatous plaque stability than degree of stenosis (Abram 2005). Consequently we should probably be less stringent in our criteria for identifying high risk patients and improvement of outcome is most likely using medical therapy to control heart rate and blood pressure, reduce inflammation, and stabilize coronary atheromatous plaques.
Beta blockers induce a shift from free fatty acids toward glucose as the main cardiac energy substrate, thus resulting in an improved energy efficiency and outcome; possess an antiarrhythmic effect and antirenin/angiotensin properties; and have antiinflammatory qualities. In addition, beta-blockade might blunt the adrenergic surge and reduce the adverse outcomes during surgery. A combination of beta blockers, low dose aspirin and statins is currently most promising and a number of large clinical trials have been looking at these treatments (Devereaux et al 2005). Statins may also reduce the incidence of stroke after carotid endarterectomy, contrast-induced nephropathy, improve graft survival, and appear to have a good safety profile. Currently statins are probably the most promising group of drugs in this perioperative setting (Biccard et al 2005).

\section{The cardiovascular protective properties of statins}

Atherosclerosis is a multi-factorial systemic disease, with hyperlipidemia as an important risk factor. For more than a decade, statins have been used clinically in lowering elevated levels of cholesterol and thus decreasing the risk for atherosclerosis (Grundy 1988; Eisenberg 1998; Ashen and Blumenthal 2005). Not only are statins highly effective in reducing low-density lipoproteins and cholesterol, they also increase high-density lipoproteins and have additional beneficial effects on systemic atherosclerosis. Statins have pleiotropic effects which improve or restore endothelial function, and can stabilize the atherosclerotic plaques by decreasing oxidative stress and vascular inflammation (Takemoto and Liao 2001; Libby 2002; Liao 2005) (Table 1).

Large multi-centre randomized controlled trials have consistently shown that statins are safe and effective in reducing low-density lipoprotein cholesterol and beneficial in the primary and secondary prevention of coronary heart

Table I Effects of statins

Lipid lowering effects
- decrease cholesterol
- increase HDL
Pleiotropic effects
- increase endothelial NO synthetase
- generation of ROS
- decrease endothelin-I production
- improve thrombogenic profile
- decrease Inflammation (decrease isoprenoid production)
- inhibition of atherosclerosis

Abberviations: CRP, C-reactive protein; HDL, high-density lipoprotein; NO, nitric oxide; ROS, reactive oxygen species. 
disease. Between 1994 and 1997, three major trials - the Scandinavian Simvastatin Survival Study (4S 1994), the CARE (Cholesterol and Recurrent Events) Trial (Sacks et al 1996) and the LIPID (Long-term Intervention with Pravastatin in Ischemic Disease) Study Group (1998) have all showed that simvastatin and pravastatin reduced the risk of a recurrent coronary event in patients with established coronary heart disease. The results of CARE and LIPID, with pravastatin, also showed that the benefits of improved survival extended to the majority of patients with coronary heart disease irrespective of their serum cholesterol levels. Since then, further studies including the MRC/BHF Heart Protection Study (Heart Protection Study Collaborative Group 2002), the ALLHAT Trial (2002), and the PROSPER Trials (Shepherd et al 2002) have also reiterated the same conclusion that statins are beneficial to patients with coronary atherosclerosis, irrespective of their cholesterol levels.

\section{Perioperative use of statins}

There are many studies involving a large number of patients to show that perioperative statin use is beneficial. Perioperative statin therapy has been shown to improve short and long-term patient outcome following cardiac and noncardiac surgery (Hindler et al 2006b). With no clinical studies reporting an increased incidence of adverse effects of perioperative statin use, it appears to be a safe and efficacious therapeutic option (Dunkelgrun 2006).

In a randomized, double-blind, placebo-controlled trial involving 6605 patients receiving a low-saturated fat, lowcholesterol diet, Downs and colleagues (1998) showed that lovastatin (20-40 mg daily) reduced the risk for the fatal or nonfatal myocardial infarction, unstable angina, or sudden cardiac death. In prospectively collected data from 1993 to 1999 involving 5052 patients undergoing percutaneous coronary angioplasty or stenting, Chan and colleagues (2002) showed that only $26.5 \%$ of the patients were treated with statins, and statin therapy was associated with a statistically significant mortality reduction at 30 days and 6 months after coronary intervention. This study was conducted in the 1990 s, and it is likely that the number of patients receiving statin therapy is much higher nowadays. Long-term statin administration was associated with a reduced incidence of adverse cardiovascular outcomes and a decreased need for coronary artery bypass graft (Chan et al 2002).

Lindenauer and colleagues (2004) performed a retrospective cohort study for a 2 year period from 2000 using data from 329 hospitals throughout the United States involving 780,591 patients who underwent major noncardiac surgery.
In this study, the overall perioperative mortality rate was $2.96 \%$, and, despite the fact that the patients on statin therapy were older, had a higher number of comorbidities, and a higher revised cardiac risk index score than those patients who were not treated, the use of statins during the early perioperative period was associated with a $1 \%$ absolute reduction of hospital mortality and a $38 \%$ reduction in the odds of inhospital mortality among patients undergoing major noncardiac surgery (Lindenaur et al 2004). Similarly, in a 10 year retrospective case-controlled study involving 2816 patients who underwent major vascular surgery between 1991 and 2000, Poldermans et al found that the risk of perioperative mortality among statin users was reduced 4.5 times compared with nonusers. This reduction in mortality was consistent in subgroup analysis independent of the type of surgery, cardiac risk factors, and cardiovascular therapy, including aspirin and $\beta$-blockers (Poldermans et al 2003). Similar conclusions were reached by Noordzij and colleagues (2007) in a casecontrolled study in 75,581 patients who underwent 108,593 noncardiac, nonvascular surgical procedures over a 10 year period between 1991 and 2001, statins were associated with a $60 \%$ mortality reduction.

In a prospective, randomized, placebo-controlled, doubleblind clinical trial involving 100 eligible consecutive patients scheduled for elective noncardiac vascular surgery in Brazil, Durazzo and colleagues (2004) found that patients taking atorvastatin had a statistically significant reduced rate of cardiac events within 6 months after surgery. In another study examining the effects of different types of statins (simvastatin, fluvastatin, pravastatin, and atorvastatin) in 211 consecutive patients who underwent major elective vascular surgery between 1998 and 2004, Schouten and colleagues (2005) found that statins were well tolerated and patients on statins had a more favorable perioperative cardiac outcome. In a 14-year period study involving 511 patients with left ventricular dysfunction undergoing noncardiac vascular surgery, Feringa and colleagues (2007) found that statins were significantly and independently associated with a reduced incidence of in-hospital cardiac related mortality. The same authors found in a cohort of 1374 patients with peripheral arterial disease that long term high dose statin therapy was associated with an improved outcome both in mortality and cardiac morbidity, irrespective of whether the patients had surgery or not (Feringa et al 2007).

Perioperative statin therapy is also associated with favorable perioperative outcomes unique to vascular surgery. Schouten and colleagues (2006c) showed that statin use can reduce the rate of growth of infrarenal aortic aneurysm, 
although better blood pressure control in statin users in this study could have been a contributing factor as significantly more statin users were on angiotensin-II-antagonist therapy. In a retrospective study involving 510 patients with a median follow-up period of 4.7 years, Kertai and colleagues (2004) showed that patients receiving both statins and betablockers had a four fold reduction in perioperative mortality and nonfatal myocardial infarction after abdominal aortic aneurysm surgery, compared with patients receiving statins alone. In a retrospective review of 446 patients who underwent infrainguinal vascular bypass with a mean follow-up period of 5.5 years, Ward and colleagues (2005) found that preoperative statin therapy was associated with less perioperative cardiac and major vascular complications, a shorter length of stay, and improved long-term survival. The findings of these studies are similar to the conclusion from the Statins for Risk Reduction in Surgery (StaRRS) study, a retrospective study with 1163 patients in which perioperative statin therapy was shown to have a highly significant myocardial protective effect in vascular patients, irrespective of their cholesterol levels (O’Neil-Callahan et al 2005). In addition, perioperative statin therapy appeared to have a renal protective effect in patients undergoing complex aortic surgery. In 77 patients with normal preoperative renal function requiring suprarenal aortic cross clamping-declamping during vascular surgery, Schouten and colleagues (2006b) showed that postoperative creatinine levels during the 30 days after surgery were significantly lower in statin users than in nonusers, and temporary postoperative hemodialysis was required in 7 patients, all of whom were not on perioperative statin therapy. There is an increasing trend towards endovascular surgery and this may involve the use of large amounts of contrast media with implications for renal function. A study of patients undergoing percutaneous coronary interventions found that pre-procedure statin use was associated with significant reduction in contrast induced nephropathy (Khanal et al 2005), although this effect is more controversial (Wong and Irwin 2007). In two recent review articles, the authors concluded that perioperative statin use in high-risk patients undergoing major elective vascular surgery is beneficial and safe, although the dosage and duration of treatment remained undefined (Boushra and Muntazar 2006; Paraskevas et al 2006).

In a study involving 98 patients who underwent carotid endarterectomy, of whom 43 (44\%) were on statin treatment at least 3 months before endarterectomy and 55 (56\%) had never received statin treatment, Koutouzis and colleagues (2007) showed that perioperative statin therapy was beneficial in that it was associated with reduced intraplaque angiogenesis in the carotid arteries. Intraplaque angiogenesis can cause progression of atherosclerotic plaque and also lead to plaque instability and rupture (Imparato et al 1983). The pathophysiology can be applied clinically as intraplaque angiogenesis and vascularization of the carotid plaque can cause ipsilateral neurological symptoms within one month of the surgical procedure and with perioperative and intraoperative evidence of distal embolization (McCarthy et al 1999). Poldermans and colleagues (2003) showed that statin use reduces perioperative cardiac and cerebrovascular mortality in patients undergoing major vascular surgery. Long term post-operative statin therapy is also beneficial as statins can enhance the rate of apoptosis in neointimal smooth muscle cells and reduce, the rate of myointimal hyperplasia and restenosis (Erl et al 2003). In a single centre study of 2127 primary carotid endarterectomy in 1853 patients between 1989 and 1999, LaMuraglia and colleagues (1995) have shown that lipid-lowering therapy is protective against carotid re-stenosis, and had favorable effects on patient survival.

In a retrospective analysis of 172 consecutive patients who underwent primary autogenous infrainguinal reconstructions with long saphenous vein, Abbruzzese and colleagues (2004) showed that statin therapy had a beneficial effect on graft patency in a follow-up period of two years. The patients on perioperative statin therapy had a much lower incidence of graft occlusions (Abbruzzese et al 2004). In 293 patients who had infrainguinal bypass grafts with either autologous vein or prosthetic conduits, Henke and colleagues (2004) showed that patients on statins have an improved graft patency rate, a decreased amputation rate and shorter in-hospital length of stay (Ward et al 2005). Higher doses of statins have additional advantage in patients with peripheral arterial disease (Feringa et al 2007).

Contrary to the peri-operative use of statins, Podermans and colleagues (2007) showed in a randomized trial that preoperative coronary revascularization in high-risk patients was not associated with an improved outcome. In order to determine the effect of statin therapy in perioperative cardiac complications and mortality in patients undergoing coronary artery bypass grafts, Collard and colleagues (2006) showed in 2666 randomized patients that preoperative statin therapy was associated with a statistically significant reduction in the risk of early cardiac related mortality. Statins did not reduce the risk of early postoperative nonfatal early cardiac complications, but early postoperative statin discontinuation was associated with increased in-hospital mortality (Collard et al 2006). 
A recent meta-analysis of 12 retrospective and 3 prospective trials with a total of 223,010 patients and showed that preoperative statin therapy was associated with $38 \%$ and $59 \%$ reduction in overall mortality after cardiac and vascular surgery (Hindler et al 2006a). In another meta-analysis of thirty-one randomized studies that compared a statin with placebo or with another statin, statin therapy was associated with a reduced relative risk of all cause mortality, cardiovascular mortality, and fatal myocardial infarction, in patients with cardiovascular disease (Ward et al 2007). However, the potential benefit of statins for low-risk populations is less clear, especially as there may be poor long-term compliance in asymptomatic younger patients.

Statins may also be an effective treatment for sepsis as a result of increased expression of endothelial nitric oxide synthase, in conjunction with down-regulation of inducible nitric oxide synthase (McGown and Brookes 2007). Combined, this results in an increase in physiological concentrations of nitric oxide, thus restoring endothelial function (Mason et al 2004; Tousoulis et al 2006). Laboratory studies have suggested that enhancement of endothelial nitric oxide synthase activity during sepsis may lead to restoration of microvascular tone, maintenance of microvascular integrity, and inhibition of cell adhesion molecules (Merx et al 2005; Xenos et al 2005). Other pleiotropic effects, including antioxidant activity and alterations in the development of vascular atherosclerosis, may also contribute to the beneficial effects of statins in this setting.

\section{Timing}

It is likely that many, if not all, patients undergoing vascular surgery could benefit from statin therapy and surgeons and anesthetists should be alerted to the importance of early identification of patients and initiation of statins, as the run-in time may affect perioperative cardiovascular outcome (Poldermans et al 2003; Durazzo et al 2004). The timing of initiation of statin therapy with respect to noncardiac surgery is yet to be defined, as there is no clear guideline on when the drugs should be started. Four weeks should allow time for a maximal clinical effect and identification of efficacy (lipid lowering) or side effects (rhabdomyolysis or raised liver enzymes) (Poldermans et al 2003). Interestingly no increased incidence of adverse effects has been reported in these trials of patients receiving perioperative statins (Landesberg et al 2003; Poldermans et al 2003). There was no statistical difference between postoperative creatine kinase levels between statin users and nonusers (Schouten et al 2005).
Postoperatively, all patients in whom long-term statin therapy is indicated should continue their therapy and any patient who has an acute coronary event postoperatively and is not on statin therapy should be considered for treatment initiation as soon as possible (Spencer et al 2004). Discontinuation of statin therapy after major vascular surgery is associated with an increased postoperative cardiac risk, suggesting that statin therapy should be continued or resumed early postoperatively (Le Manach et al 2007). It would be reasonable to continue statins for at least 72 hours postoperatively as this is the time period most likely to be associated with an acute coronary event (Badner et al 1998). Temporarily discontinuing therapy for a day appears to be safe (Schouten et al 2005), but high-risk patients are probably at increased risk after three days (Heeschen et al 2003). It is likely that continued statin administration will improve outcome further, as in patients who experience acute coronary syndromes, the outcome continues to improve with prolonged statin administration (Eagle et al 1997; Aronow et al 2001), and vascular patients probably benefit from long-term statin therapy anyway (Newby and Nimmo 2004; Feringa et al 2006).

\section{Conclusion}

There is growing evidence in the world literature to suggest that perioperative statin therapy is cost-effective in reducing morbidity and mortality in elective vascular surgery. In a pharmaco-economic analysis of two prospective studies examining the utility of statins in the perioperative period in patients undergoing major vascular surgery, Biccard and colleagues (2005) showed the number-needed-to-treat to prevent an adverse cardiac event was fifteen. Statins may be under-prescribed in vascular surgery patients, especially since many of them have clinically occult cardiac ischemia.

Despite a number of interesting potential benefits and a good safety profile, there is still a lack of large multicenter level I evidence to support the routine use of statins during noncardiac surgery, and most of the evidence is from retrospective cohort studies (Bolsin et al 2007). Although arguments based on biologic plausibility, cohort series, small randomized trials and extrapolation from nonsurgical cohorts may be misleading, there is already overwhelming evidence of efficacy. A large multi-centre randomized control trial on perioperative statin use may be difficult to conduct as there is overwhelming evidence to suggest that statins have a beneficial cardiovascular protective effect, whether the patient is undergoing surgery or not. Some authors may consider it unethical to with-hold statin treatment to conduct 
such a trial. Others have shown that in order to reduce the perioperative risk of myocardial events by $25 \%$, at least 6000 patients would need to be recruited for such a study (Kapoor et al 2006). Up to now, there is no consensus to support a role for the routine administration in patients without established coronary artery disease undergoing noncardiac surgery; although some have suggested that perioperative statin therapy for patients undergoing vascular surgery may present the most cost-effective use of statin therapy yet described (Biccard et al 2005). Rather to examine the perioperative use of statin, future trials may be aimed at determining the optimal duration and dosage of perioperative statin therapy (Paraskevas et al 2006).

\section{References}

Abbruzzese TA, Havens J, Belkin M, et al. 2004. Statin therapy is associated with improved patency of autogenous infrainguinal bypass grafts. J Vasc Surg, 39:1178-85.

Abrams J. 2005. Clinical practice. Chronic stable angina. $N$ Engl J Med, 352:2524-33.

Adesanya AO, de Lemos JA, Greilich NB, et al. 2006. Management of perioperative myocardial infarction in noncardiac surgical patients. Chest, 130:584-96.

ALLHAT. 2002. Major outcomes in moderately hypercholesterolemic, hypertensive patients randomized to pravastatin vs usual care: the Antihypertensive and Lipid-Lowering Treatment to Prevent Heart Attack Trial (ALLHAT-LLT). JAMA, 288:2998-3007.

Aronow HD, Topol EJ, Roe MT, et al. 2001. Effect of lipid-lowering therapy on early mortality after acute coronary syndromes: an observational study. Lancet, 357:1063-8.

Ashen MD, Blumenthal RS. 2005. Clinical practice. Low HDL cholesterol levels. N Engl J Med, 353:1252-60.

Ashton CM, Petersen NJ, Wray NP, et al. 1993. The incidence of perioperative myocardial infarction in men undergoing noncardiac surgery. Ann Intern Med, 118:504-10.

Badner NH, Knill RL, Brown JE, et al. 1998. Myocardial infarction after noncardiac surgery. Anesthesiology, 88:572-8.

Bayly PJM, Matthews JNS, Dobson PM. et al. 2001. In-hospital mortality from abdominal aortic surgery in Great Britain and Ireland: Vascular Anaesthesia Society audit. BJS, 88:687-92.

Biccard BM, Sear JW, Foëx P. 2005. The pharmaco-economics of perioperative statin therapy. Anaesthesia, 60:1059-63.

Boden WE, O'Rourke RA, Teo KK, et al. 2007. COURAGE Trial Research Group. Optimal medical therapy with or without PCI for stable coronary disease. N Engl J Med, 356:1503-16.

Bolsin S, Colson M, Conroy M. 2007. Beta-blockers and statins in noncardiac surgery. BMJ, 334:1283-4.

Boushra NN, Muntazar M. 2006. Review article: The role of statins in reducing perioperative cardiac risk: Physiologic and clinical perspectives. Can J Anaesth, 53:1126-47.

Chan AW, Bhatt DL, Chew DP, et al. 2002. Early and sustained survival benefit associated with statin therapy at the time of percutaneous coronary intervention. Circulation, 105:691-6.

Cohen MC, Aretz TH. 1999. Histological analysis of coronary artery lesions in fatal postoperative myocardial infarction. Cardiovasc Pathol, 8:133-9.

Collard CD, Body SC, Shernan SK, et al; Multicenter Study of Perioperative Ischemia (MCSPI) Research Group, Inc; Ischemia Research and Education Foundation (IREF) Investigators. 2006. Preoperative statin therapy is associated with reduced cardiac mortality after coronary artery bypass graft surgery. J Thorac Cardiovasc Surg, 132:392-400.
Devereaux PJ, Beattie WS, Choi PT, et al. 2005. How strong is the evidence for the use of perioperative beta blockers in non-cardiac surgery? Systematic review and meta-analysis of randomised controlled trials. BMJ, 331:313-21.

Downs JR, Clearfield M, Weis S, et al. 1998. Primary prevention of acute coronary events with lovastatin in men and women with average cholesterol levels: results of AFCAPS/TexCAPS. Air Force/Texas Coronary Atherosclerosis Prevention Study. JAMA, 279:1615-22.

Dunkelgrun M, Schouten O, Feringa HH, et al. 2006. Beneficial effects of statins on perioperative cardiovascular outcome. Curr Opin Anaesthesiol, 19:418-22.

Durazzo AE, Machado FS, Ikeoka DT, et al. 2004. Reduction in cardiovascular events after vascular surgery with atorvastatin: a randomized trial. J Vasc Surg, 39:967-75.

Eagle KA, Rihal CS, Mickel MC, et al. 1997. Cardiac risk of noncardiac surgery: influence of coronary disease and type of surgery in 3,368 operations. CASS Investigators and University of Michigan Heart Care Program. Coronary Artery Surgery Study. Circulation, 96:1882-7.

Eisenberg DA. 1998. Cholesterol lowering in the management of coronary artery disease: the clinical implications of recent trials. Am J Med, 104(2A):2S-5S

Erl W, Hristov M, Neureuter M, et al. 2003. HMG-CoA reductase inhibitors induce apoptosis in neointima-derived vascular smooth muscle cells, Atherosclerosis, 169:251-8.

Feringa HH, Bax JJ, Schouten O, et al. 2006. Protecting the heart with cardiac medication in patients with left ventricular dysfunction undergoing major noncardiac vascular surgery. Semin Cardiothorac Vasc Anesth, 10:25-31.

Feringa HH, Karagiannis SE, van Waning VH, et al. 2007. The effect of intensified lipid-lowering therapy on long-term prognosis in patients with peripheral arterial disease. J Vasc Surg, 45:936-43.

Gibson CM, Ciaglo LN, Southard MC, et al. 2007. Diagnostic and prognostic value of ambulatory ECG (Holter) monitoring in patients with coronary heart disease: a review. J Thromb Thrombolysis, 23:135-45.

Grundy SM. 1988. HMG-CoA reductase inhibitors for treatment of hypercholesterolemia. N Engl J Med, 319:24-33.

Heart Protection Study Collaborative Group. 2002. MRC/BHF Heart Protection Study of antioxidant vitamin supplementation in 20,536 high-risk individuals: a randomised placebo-controlled trial. Lancet 2002; 360:23-33

Heeschen C, Hamm CW, Laufs U, et al. 2003. Withdrawal of statins in patients with acute coronary syndromes. Circulation, 107:e27.

Henke PK, Blackburn S, Proctor MC, et al. 2004. Patients undergoing infrainguinal bypass to treat atheroscle-rotic vascular disease are underprescribed cardio-protective medications: effect on graft patency, limb salvage, and mortality. J Vasc Surg, 39:357-65.

Hindler K, Eltzschig HK, Fox AA, et al. 2006a. Influence of statins on perioperative outcomes. J Cardiothorac Vasc Anesth, 20:251-8.

Hindler K, Shaw AD, Samuels J, et al. 2006b. Improved postoperative outcomes associated with preoperative statin therapy. Anesthesiology, 105:1260-72.

Imparato AM, Riles TS, Mintzer R, et al. 1983. The importance of hemorrhage in the relationship between gross morphologic characteristics and cerebral symptoms in 376 carotid artery plaques, Ann Surg, 197:195-203.

Kapoor AS, Kanji H, Buckingham J, et al. 2006. Strength of evidence for perioperative use of statins to reduce cardiovascular risk: systematic review of controlled studies. BMJ, 333:1149.

Kertai MD, Boersma E, Westerhout CM, et al. 2004. A combination of statins and beta-blockers is independently associated with a reduction in the incidence of perioperative mortality and nonfatal myocardial infarction in patients undergoing abdominal aortic aneurysm surgery. Eur J Vasc Endovasc Surg, 28:343-52.

Kertai MD, Bogar L, Gal J, et al. 2006. Pre-operative coronary revascularization: an optimal therapy for high-risk vascular surgery patients? Acta Anaesthesiol Scand, 50:816-27. 
Khanal S, Attallah N, Smith DE, et al. 2005. Statin therapy reduces contrast-induced nephropathy: an analysis of contemporary percutaneous interventions. Am J Med, 118:843-9.

Koutouzis M, Nomikos A, Nikolidakis S, et al. 2007. Statin treated patients have reduced intraplaque angiogenesis in carotid endarterectomy specimens. Atherosclerosis, 192:457-63.

LaMuraglia GM, Stoner MC, Brewster DC, et al. 2005. Determinants of carotid endarterectomy anatomic durability: effects of serum lipids and lipid-lowering drugs. J Vasc Surg, 41:762-8.

Landesberg G, Mosseri M, Wolf YG, et al. 2003. Preoperative thallium scanning, selective coronary revascularization, and long-term survival after major vascular surgery. Circulaton, 108:177-83.

Le Manach Y, Godet G, Coriat P, et al. 2007. The impact of postoperative discontinuation or continuation of chronic statin therapy on cardiac outcome after major vascular surgery. Anesth Analg, 104:1326-33.

Liao JK. 2005. Clinical implications for statin pleiotropy. Curr Opin Lipidol, 16:624-9.

Libby P. 2002. Inflammation in atherosclerosis. Nature, 420:868-74.

Lindenauer PK, Pekow P, Wang K, et al. 2004. Lipid-lowering therapy and in-hospital mortality following major noncardiac surgery. JAMA, 291:2092-9.

LIPID Study Group. 1998. Prevention of cardiovascular events and death with pravastatin in patients with coronary heart disease and a broad range of initial cholesterol levels. The Long-Term Intervention with Pravastatin in Ischaemic Disease (LIPID) Study Group. $N$ Engl J Med, 339:1349-57.

Mangano DT, Browner W, Hollenberg M, et al; for the Study of Perioperative Ischemia Research Group. 1992. Long-term cardiac prognosis following noncardiac surgery. JAMA, 268:233-9.

Mason RP, Walter MF, Jacob RF. 2004. Effects of HMG-CoA reductase inhibitors on endothelial function: role of microdomains and oxidative stress. Circulation, 109(21 Suppl 1):II34-II41.

McCarthy MJ, Loftus IM, Thompson MM, et al. 1999. Angiogenesis and the atherosclerotic carotid plaque: an association between symptomatology and plaque morphology. J Vasc Surg, 30:261-8.

McGown CC, Brookes ZL. 2007. Beneficial effects of statins on the microcirculation during sepsis: the role of nitric oxide. Br J Anaesthsia, 98:163-75.

Merx MW, Liehn EA, Graf J, et al. 2005. Statin treatment after onset of sepsis in a murine model improves survival. Circulation, 112:117-24.

Newby DE, Nimmo AF. 2004. Prevention of cardiac complications of noncardiac surgery: stenosis and thrombosis. Br J Anaesth, 92:628-32.

Noordzij PG, Poldermans D, Schouten O, et al. 2007. Beta-blockers and statins are individually associated with reduced mortality in patients undergoing noncardiac, nonvascular surgery. Coron Artery Dis, 8:67-72.

O’Neil-Callahan K, Katsimaglis G, Tepper MR, et al. 2005. Statins decrease perioperative cardiac complications in patients undergoing noncardiac vascular surgery: the Statins for Risk Reduction in Surgery (StaRRS) study. J Am Coll Cardiol, 45:336-42.

Paraskevas KI, Liapis CD, Hamilton G, et al. 2006. Can statins reduce perioperative morbidity and mortality in patients undergoing non-cardiac vascular surgery? Eur J Vasc Endovasc Surg, 32:286-93.

Pasternack PF, Grossi EA, Baumann FG, et al. 1989. The value of silent myocardial ischemia monitoring in the prediction of perioperative myocardial infarction in patients undergoing peripheral vascular surgery. J Vasc Surg, 10:617-25.

Poldermans D, Bax JJ, Kertai MD, et al. 2003. Statins are associated with a reduced incidence of perioperative mortality in patients undergoing major noncardiac vascular surgery. Circulation, 107:1848-51.

Poldermans D, Bax JJ, Kertai MD, et al. 2003. Statins are associated with a reduced incidence of perioperative mortality in patients undergoing major non-cardiac vascular surgery. Circulation, 107:1848-51.

Poldermans D, Boersma E, Bax JJ, et al. 2001. Correlation of location of acute myocardial infarct after noncardiac vascular surgery with preoperative dobutamine echocardiographic findings. Am J Cardiol, $88: 1413-14$
Poldermans D, Schouten O, Vidakovic R, et al.; DECREASE Study Group. 2007. A clinical randomized trial to evaluate the safety of a noninvasive approach in high-risk patients undergoing major vascular surgery: the DECREASE-V Pilot Study. J Am Coll Cardiol, 49:1763-9.

Priebe HJ. 2004. Triggers of perioperative myocardial ischaemia and infarction. Br J Anaesth, 93:9-20.

[4S] Scandinavian Simvastatin Study Group. 1994. Randomised trial of cholesterol lowering in 4444 patients with coronary heart disease: the Scandinavian Simvastatin Survival Study (4S). Lancet, 344:1383-9.

Sacks FM, Pfeffer MA, Moye LA, et al. 1996. The effect of pravastatin on coronary events after myocardial infarction in patients with average cholesterol levels. Cholesterol and Recurrent Events Trial investigators. N Engl J Med, 335:1001-9.

Schouten O, Bax JJ, Dunkelgrun M, et al. 2006a. Statins for the prevention of perioperative cardiovascular complications in vascular surgery. J Vasc Surg, 44:419-24.

Schouten O, Kertai MD, Bax JJ, et al. 2005. Safety of perioperative statin use in high-risk patients undergoing major vascular surgery. Am J Cardiology, 95:658-60.

Schouten O, Kok NF, Boersma E, et al. 2006b. Effects of statins on renal function after aortic cross clamping during major vascular surgery. $\mathrm{Am}$ J Cardiol, 97:1383-5.

Schouten O, van Laanen JHH, Boersma E, et al. 2006c. Statins are associated with a reduced infrarenal abdominal aortic aneurysm growth. Eur J Vasc Endovasc Surg, 32:21-6.

Shepherd J, Blauw GJ, Murphy MB, et al. 2002. Pravastatin in elderly individuals at risk of vascular disease (PROSPER): a randomised controlled trial. Lancet, 360:1623-30.

Spencer FA, Allegrone J, Goldberg RJ, et al; GRACE Investigators. 2004. Association of statin therapy with outcomes of acute coronary syndromes: the GRACE study. Ann Intern Med, 140:857-66.

Takemoto M, Liao JK. 2001. Pleiotropic effects of 3-hydroxy-3-methylglutaryl coenzyme a reductase inhibitors. Arterioscler Thromb Vasc Biol, 21:1712-19.

Tousoulis D, Antoniades C, Koumallos N, et al. 2006. Novel therapies targeting vascular endothelium. Endothelium, 13:411-21.

Ward RP, Leeper NJ, Kirkpatrick JN, et al. 2005. The effect of preoperative statin therapy on cardiovascular outcomes in patients undergoing infrainguinal vascular surgery. Int J Cardiol, 104:264-8.

Ward S, Lloyd Jones M, Pandor A, et al. 2007. A systematic review and economic evaluation of statins for the prevention of coronary events. Health Technol Assess, 11:1-178.

Wong GTC, Irwin MG. 2007. Contrast induced nephropathy. Br J Anaesth, 99:474-83.

Xenos ES, Stevens SL, Freeman MB, et al. 2005. Nitric oxide mediates the effect of fluvastatin on intercellular adhesion molecule-1 and platelet endothelial cell adhesion molecule-1 expression on human endothelial cells. Ann Vasc Surg, 19:386-92.

Zaman AG, Helft G, Worthley SG, et al. 2000. The role of plaque rupture and thrombosis in coronary artery disease. Atherosclerosis, 149:251-66. 
